\title{
Moving average filters and unit roots *
}

\author{
Philip Hans Franses \\ Econometric Institute, Erasmus University Rotterdam, 3000 DR Rotterdam, Netherlands \\ Received 26 June 1991 \\ Accepted 17 October 1991
}

Moving average filtering a stationary AR(1) time series yields higher valued first order autocorrelations. Its implications for unit root testing in seasonally (un-) adjusted time series are evaluated theoretically, via simulations, as well as with an empirical example.

\section{Introduction}

Testing for unit roots in economic time series has now become standard practice in empirical research. These series are, however, not always used in their raw form, but are often considered after some transformations. One such transformation is to apply a moving average filter to the original time series. A property of such a filter is that is smoothens the series, and hence one might expect that the occurence of a unit root becomes more likely. Indeed, as, e.g., Howrey (1968) already showed, cycles may become longer in such filtered series. Since a moving average filter is also used in most seasonal adjustment procedures, it is likely that one may find more evidence for the presence of unit roots in seasonally adjusted series than in the unadjusted original series [see Bhargava (1990), Ghysels (1990), Ghysels and Perron (1990), and Jaeger and Kunst (1990)].

In this letter, I consider the application of a moving average filter to an $\mathrm{AR}(1)$ generated series in more detail. This AR(1) process is chosen for expository purposes, but an extension to higher order AR processes is straightforward, as will be pointed out briefly. In section 1, it is shown that the autocorrelations of the filtered series exceed those of the original series in case the AR(1) process is stationary. On the other hand, in case the generating series has a unit root, the filtered series also contains a unit root. In section 2, the implications of this result are discussed for unit root testing in seasonal time series. The test procedure of Hylleberg et al. (1990) for seasonally unadjusted series wi'l also be evaluated. An empirical example as well as some simulation evidence are used to emphasize the arguments. The final section contains some concluding remarks.

\section{Autocorrelations}

Consider the AR(1) model for a zero mean process $y_{t}$,

$$
y_{t}=\phi y_{t-1}+\epsilon_{t}
$$

\footnotetext{
* Thanks are due to Eric Ghysels and Svend Hylleberg for helpful discussions.
} 
where $\epsilon_{t}$ is an uncorrelated zero mean variable with variance $\sigma_{\epsilon}^{2}$, and for which it is assumed for convenience that $\phi \in[0,1]$, since these parameter values are also often of practical interest. The autocovariances of (1) are defined by $\gamma_{k y}=\phi^{k} \sigma_{\epsilon}^{2} /\left(1-\phi^{2}\right)$, and its autocorrelations by $\rho_{k y}=\phi^{k}$, where $k$ is $0,1,2, \ldots$ Consider further a moving average filtered process $x_{t}$ given by

$$
x_{t}=y_{t}+y_{t-1}
$$

where a division by 2 is deleted since the focus is on autocorrelations. It is easy to see that for $x_{t}$ applies that its autocovariances are given by

$$
\begin{aligned}
& \gamma_{0 x}=2 \gamma_{0 y}+2 \gamma_{1 y}, \\
& \gamma_{1 x}=\gamma_{0 y}+2 \gamma_{1 y}+\gamma_{2 y}, \\
& \gamma_{k x}=\gamma_{k-1 y}+2 \gamma_{k y}+\gamma_{k+1 y}, \text { for } k=2,3, \ldots
\end{aligned}
$$

such that $\rho_{0 x}=1, \rho_{1 x}=(\phi+1) / 2$, and $\rho_{k x}=\phi^{k-1} \rho_{1 x}$ for $k>1$. Comparing the autocorrelations of (1) and (2) gives that for $k=1,2, \ldots$,

$$
\begin{aligned}
& \rho_{k x}>\rho_{k y} \text { if } \phi \in[0,1), \\
& \rho_{k x}=\rho_{k y} \text { if } \phi=1 .
\end{aligned}
$$

Moreover note that $\rho_{1 x}=0.5$ if $\phi=0$. Of course, the result in (7) could already be seen from (2).

To illustrate that an extension to higher order processes is fairly straightforward, consider the $\mathrm{AR}(2)$ process $y_{t}=\phi_{1} y_{t-1}+\phi_{2} y_{t-2}+\epsilon_{t}$ for which it holds that $\rho_{1 y}=\phi_{1} /\left(1-\phi_{2}\right)$. One assumption for stationarity is that $-1<\phi_{2}<1$. The first order autocorrelation of $x_{t}$, when $x_{t}$ is again defined as in (2), can be found from (3) and (4). When $\rho_{2 y}$ is replaced by $\phi_{1} \rho_{1 y}+\phi_{2}$, it is given by $\rho_{1 x}=\left(1+2 \rho_{1 y}+\phi_{1} \rho_{1 y}+\phi_{2}\right) /\left(2+2 \rho_{1 y}\right)$. It is now easy to derive that

$$
\begin{aligned}
\rho_{1 x}-\rho_{1 y} & =\left(1+\phi_{1} \rho_{1 y}-2 \rho_{1 y}^{2}+\phi_{2}\right) /\left(2+2 \rho_{1 y}\right), \\
& =\left(\left(1+\phi_{2}\right)\left(1-\rho_{1 y}^{2}\right)\right) /\left(2+2 \rho_{1 y}\right),
\end{aligned}
$$

which is zcro in case $\rho_{1 y}=1$, and excecds zero in the stationary case. In summary, when an AR series $y_{t}$ has a unit root, i.e. when it is $I(1)$, then $x_{t}$ is also $I(1)$. When $y_{t}$ is a stationary AR process, the $x_{t}$ is stationary too, albeit with higher valued autocorrelations. An implication is that the size of any test procedure for the null hypothesis of a unit root will not be affected by an application of a moving average filter, but the rejection rate under the alternative hypothesis will become lower for the averaged series.

It is easy to see that such an argument applies a fortiori to moving averages including more terms. For example, consider $z_{t}=\sum_{i=1}^{m} \epsilon_{t-i+1}$, for which it applies that $\rho_{k z}=1-k / m$, for $k=1,1$, $2, \ldots, m-1$, and 0 for $k=m, m+1, \ldots$ So, averaging, e.g., ten white noise variables yields a $\rho_{1}$ equal to 0.9 .

A commonly applied filter in seasonal time series, such as in seasonal adjustment procedures, is the seasonal moving average filter, which for quarterly data is given by $s_{t}=\left(1+B+B^{2}+B^{3}\right) y_{t}$, where $B^{k} y_{t} \equiv y_{t-k}$. When $y_{t}$ is again the AR(1) process as in (1), similar results as in (6) and (7) 
can be derived for $s_{t}$. It is clear that for monthly data the seasonal moving average filtered series has even higher valued autocorrelations.

\section{Implications}

These results have several implications for testing for unit roots in seasonally (un-)adjusted series. The moving average filter $s_{t}$ is often applied in seasonal adjustment procedures for quarterly time series. In case the underlying process is $\operatorname{AR}(1)$ with a unit root, the moving average filtered series also contains a unit root. Hence, the size of unit root tests is not affected. When the AR(1) process is stationary, the autocorrelations of the filtered series are higher than those of the original series. The rejection rate under the alternative hypothesis of unit root tests will therefore become lower. The empirical occurrence of such power loss for seasonally adjusted time series is demonstrated in Ghysels (1990), Ghysels and Perron (1990) and Jaeger and Kunst (1990). Indeed, this loss appears to be larger for monthly adjusted series than for quarterly series, see Ghysels (1990).

A test procedure for unit roots in seasonally unadjusted quarterly series is proposed in Hylleberg et al. (1990) [HEGY4]. Their test equation considers the auxiliary regression

$$
\varphi^{*}(B) y_{4 t}=\mu_{t}+\pi_{1} y_{1 t-1}+\pi_{2} y_{2 t-1}+\pi_{3} y_{3 t-2}+\pi_{4} y_{3 t-1}+\epsilon_{t},
$$

where $y_{1 t}=\left(1+B+B^{2}+B^{3}\right) y_{t}, \quad y_{2 t}=-(1-B)\left(1+B^{2}\right) y_{t}, \quad y_{3 t}=-\left(1-B^{2}\right) y_{t}, \quad$ and $y_{4 t}=(1-$ $\left.B^{4}\right) y_{t}$, and where $\mu_{t}$ can include deterministic elements like a constant, seasonal dummies and a trend. The significance of the $\pi_{i}$ parameters in (8) establishes the presence or absence of a nonseasonal unit root $\left(\pi_{1}\right)$, since $\left(1-B^{4}\right)$ is equal to $(1-B)\left(1+B+B^{2}+B^{3}\right)$, and of seasonal unit roots $\left(\pi_{2}, \pi_{3}\right.$ and $\left.\pi_{4}\right)$. [See Osborn (1990) and Otto and Wirjanto (1990) for applications.]

Suppose for the moment that a stationary AR(1) process as in (1) is the data generating process. When applying the HEGY4 test to such a series one would expect that all $\pi_{i}$ are significantly different from zero, and that the $\pi_{i}$ related to the seasonal unit roots establish that the residuals of (8) appear to be uncorrelated. However, the moving average filter in the $y_{1 t}$ variable may cause that the null hypothesis of a nonseasonal unit root is spuriously accepted.

An illustrative example of this occurrence is given by the $\log$ of the U.K. earnings series, say $E_{t}$, as it is considered in Osborn (1990). When the HEGY4 procedure is applied to the first differenced series to test for $I(2)$ patterns, the $t$ value for the $\hat{\pi}_{1}$ is -2.44 , which is insignificant. On the other hand, when the $E_{t}$ series is evaluated with the HEGY4 method, the $t$ value for the $\hat{\pi}_{1}$ is -1.92 . This conflicting outcome is however caused by the moving average filter in the first regression, since an augmented Dickey-Fuller regression for the first differenced series yields a highly significant $t$ value of -4.880 . This small example illustrates that after the application of the HEGY method, an additional step, which is to apply a simple Dickey-Fuller regression, seems useful.

The power of the HEGY test procedure for a nonseasonal unit root can thus be low in case a stationary AR process is the underlying process. Moreover, it can be expected that this power may become lower in case the HEGY procedure is applied to monthly series, as is done in, e.g., Franses (1990,1991) [HEGY12] and Beaulieu and Miron (1991). A small simulation experiment illustrates this phenomenon. The data generating process is an AR(1) process as in (1) with parameter values 0.8 and 1.0 and $y_{0}=0$, for which in 100 replications 144 observations are generated. The first 24 are discarded to reduce any starting-up effects. The remaining 120 observations are treated as being nonseasonal, quarterly and monthly series, and the conventional Dickey-Fuller procedure [DF], and the HEGY4 and HEGY12 methods are applied, respectively. The HEGY methods both assume that $\varphi^{*}(B)$, see (8), can be set equal to 1 . All regressions are checked for eventual residual 
Table 1

Rejection frequencies of tests for nonseasonal unit roots DGP is $y_{t}=\alpha y_{t-1}+\epsilon_{t}, \epsilon_{t} \sim \mathrm{N}(0,1), 120$ observations, 100 replications

\begin{tabular}{lllll}
\hline$\alpha$ & Size & \multicolumn{2}{l}{ Test procedures $^{\text {a }}$} & HEGY12 \\
\cline { 3 - 5 } & & DF & HEGY4 & 0.41 \\
0.8 & 0.05 & 0.94 & 0.82 & 0.61 \\
& 0.10 & 1.00 & 0.93 & 0.02 \\
1.0 & 0.05 & 0.04 & 0.02 & 0.05 \\
& 0.10 & 0.07 & 0.04 & .0. \\
\hline
\end{tabular}

a The test procedures are the Dickey-Fuller $\tau_{\mu}$-test statistic (DF), the Hylleberg et al. (1990) procedure for quarterly time series (HEGY4), and the latter method when it is extended to monthly time series (HEGY12).

autocorrelations, but almost all of them seem to be free from such correlations. The auxiliary regressions contain no trend, but do contain a constant since one usually does so in practice. The $5 \%$ and $10 \%$ critical values for the relevant test statistics are -2.89 and -2.58 for DF, -2.88 and -2.58 for HEGY4, and -2.69 and -2.40 for HEGY12. The results are displayed in table 1 . It is clear that the size of the test procedures does not seem to be affected, but that the power becomes lower in case the regressor which is related to the presence of a nonseasonal unit root averages more terms.

\section{Concluding remarks}

It is shown that moving average filtered series have higher valued autocorrelations than those of an underlying stationary AR(1) process. This may establish that testing for unit roots in, e.g., seasonal time series should preferably be considered for seasonally unadjusted series since most adjustment procedures include such a moving average filter.

An application of the Hylleberg et al. (1990) test procedure may be suitable for the unadjusted series. An additional step in this method can however be useful, since testing for a nonseasonal unit root in the original series is carried out by testing for a unit root in a moving average filtered series. One suggestion is to first test for the presence of seasonal unit roots, and when these appear to be absent, to test for a nonseasonal unit root in a conventional Dickey-Fuller regression.

\section{References}

Beaulieu, J.J. and J.A. Miron, 1991, Seasonal unit roots in aggregate U.S. data, Unpublished manuscript.

Bhargava, A., 1990, An econometric analysis of the U.S. postwar G.N.P., Journal of Population Economics 3, $147-156$.

Franses, P.H., 1990, Testing for seasonal unit roots in monthly data, Econometric Institute Report 9032/A (Erasmus University Rotterdam, Rotterdam).

Franses, P.H., 1991, Seasonality, non-stationarity and the forecasting of monthly time series, International Journal of Forecasting 7.

Ghysels, E, 1990, Unit-root tests and the statistical pitfalls of seasonal adjustment: The case of U.S. postwar real gross national product, Journal of Business \& Econometric Statistics 8, 145-152.

Ghysels, E. and P. Perron, 1990, The effect of seasonal adjustment filters on tests for a unit root, Unpublished manuscript (C.R.D.E. Montreal, Montreal, Ont.),

Howrey, E.Ph., 1968, A spectrum analysis of the long-swing hypothesis, International Economic Review 9, $228-252$.

Hylleberg, S., R.F. Engle, C.W.J. Granger and B.S. Yoo, 1990, Seasonal integration and cointegration, Journal of Econometrics 44, 215-238. 
Jaeger, A. and R.M. Kunst, 1990, Seasonal adjustment and measuring persistence in output, Journal of Applied Econometrics $5,47-58$.

Osborn, D.R., 1990, A survey of seasonality in UK macroeconomic variables, International Journal of Forecasting 6, $327-336$.

Otto, G. and T. Wirjanto, 1990, Seasonal unit-root tests on Canadian macroeconomic time series, Economics Letters 34 , $117-120$. 